\title{
Porcine JAB1 significantly enhances apoptosis induced by staurosporine
}

\author{
P Jiang', J Wang ${ }^{1}$, Z Kang ${ }^{1}$, D Li ${ }^{1}$ and D Zhang ${ }^{*, 1}$
}

c-Jun activation domain-binding protein-1 (JAB1), also known as the subunit 5 of the COP9 signalosome, is a multifunctional protein that regulates cell proliferation, apoptosis and oncogenesis by interacting with and subsequently degrading a large number of proteins. Although human JAB1 (hJAB1) has been studied for a long time, studies on porcine JAB1 (pJAB1) have never been reported. In the present study, we cloned and characterized the pJAB1 gene. The genomic structure of the pJAB1 gene was determined. The open-reading frame of pJAB1 encoded 334 amino acids. The deduced amino acid sequence was highly similar to homologs in other species. Furthermore, the tertiary structure analysis and phylogenetic analysis indicated that JAB1 was highly conservative among species. pJAB1 may interact with several proteins according to protein-protein interactions analysis. In addition, pJAB1 was found to be universally expressed in porcine tissues. Subcellular localization analysis showed that GFP-pJAB1 fusion protein distributed specifically in the cytoplasm. Flow cytometric analysis proved that pJAB1 significantly enhanced apoptosis induced by staurosporine, which at least partially depended on the activation of caspase-9 and caspase-3. This study is useful for understanding the function of pJAB1 and offers a potential molecular model for the investigation of diseases related to hJAB1.

Cell Death and Disease (2013) 4, e823; doi:10.1038/cddis.2013.357; published online 3 October 2013

Subject Category: Cancer

C-Jun activation domain-binding protein-1 (JAB1), also known as the subunit 5 of the COP9 signalosome, was first identified by Claret et al. ${ }^{1}$ through a two-hybrid screen using the c-Jun $\mathrm{N}$-terminal activation domain as a bait. As a critical regulator of cell survival and proliferation, JAB1 is evolutionarily conserved among plants, fission yeast, mice and humans. ${ }^{2-4}$ Previous reports show that JAB1 is a multifunctional protein that regulates cell proliferation, apoptosis and oncogenesis by interacting with and subsequently degrading a large number of proteins such as the Brain-specific kinase 2, the cyclindependent kinase inhibitor p27 ${ }^{\mathrm{kip} 1}, \mathrm{p} 53$, Smad4 and the capsid of West Nile virus. ${ }^{5-9}$ In addition, as a key CSN subunit, JAB1 can integrate multiple functions of the CSN complex. ${ }^{10}$ For instance, JAB1 contains an Mpr1-Pad1-N-terminal (MPN) domain metalloenzyme motif that is essential for the CSN isopeptidase activity responsible for the deneddylation of the cullin-RING ubiquitin ligases by CSN. ${ }^{11-14}$ Therefore, JAB1 exists not only as a member of the CSN complex but also as a monomer with a number of different unique protein interactions and functions outside of the CSN. Recently, JAB1 has been shown to be involved in diverse cancers, such as pancreatic adenocarcinoma, breast carcinoma and hepatocellular carcinoma. ${ }^{15-17}$ However, the specific role of JAB1 and its cellular mechanism in oncogenic processes still remain obscure.
Staurosporine (STS), which is an inhibitor of protein kinase $\mathrm{C}$ (PKC) and an activator of the mitochondrion-mediated apoptotic pathway, ${ }^{18}$ can induce apoptosis of diverse cell lines, such as NIH-3T3 cells, ${ }^{19}$ rat astrocytes, ${ }^{20}$ HeLa Cells, ${ }^{21} 661 \mathrm{w}$ cells, ${ }^{22}$ SH-SY5Y cells, ${ }^{23}$ SMMC-7721 and BEL-7404 cells. ${ }^{24}$ A majority of these cell lines are cancer cells, which suggests that STS may inhibit growth of tumors. Apoptosis induced by STS can be positively regulated by $\mathrm{PKC}^{25}$ and membrane-associated guanylate kinase inverted $2,{ }^{26}$ and be negatively regulated by phosphoinositide 3-kinase, ${ }^{25}$ FK506 binding protein 51 (Daudt and Yorio ${ }^{22}$ ) and axin. ${ }^{27}$ However, there is no report whether JAB1 is involved in apoptosis induced by STS.

Similar to humans, pigs are also an outbred species, and they are physiologically, anatomically and immunologically similar to humans. ${ }^{28-31}$ Recently, pigs were reported to be used as medical models for studying several human diseases such as sepsis, ${ }^{32}$ abdominal aortic aneurysm, ${ }^{33}$ severe tension pneumothorax ${ }^{34}$ and many kinds of cancers. ${ }^{35}$ Therefore, pigs are optimal animals used in scientific research.

Although the function of human JAB1 (hJAB1) has been studied for a long time, porcine JAB1 (pJAB1) has little been studied. Does pJAB1 have similar structures and have a role similar to its homolog in human? Is pJAB1 involved in apoptosis induced by STS? To answer these questions, in this study, we cloned the $p J A B 1$ gene, characterized its

\footnotetext{
${ }^{1}$ College of Veterinary Medicine, Northwest A\&F University, 22 Xinong Road, Yangling District, Xi'an City, China

*Corresponding author: D Zhang, College of Veterinary Medicine, Northwest A\&F University, 22 Xinong Road, Yangling District, Xi'an City 712100, China. Tel: + 86298709 1117; Fax: + 86298709 1032; E-mail: zhangdeli@ tsinghua.org.cn

Keywords: porcine JAB1; bioinformatics analysis; spatial expression; subcellular localization; enhancement to apoptosis

Abbreviations: JAB1, c-Jun activation domain-binding protein-1; pJAB1, porcine JAB1; MPN, Mpr1-Pad1-N-terminal; STS, staurosporine; PKC, protein kinase C; hJAB1, human JAB1; Mw, molecular weight; pl, isoelectric point; COPS, COP9 constitutive photomorphogenic homolog subunit; TP53, tumor protein p53; PSMD, proteasome 26S subunit non-ATPase; GFER, growth factor augmenter of liver regeneration; RNF139, ring finger protein 139; GO, Gene Ontology; SUVEC, the established swine umbilical vein endothelial cell line; PK15, porcine kidney-15 cell line; FCM, flow cytometry; HRP, horseradish peroxidase

Received 29.5.13; revised 08.8.13; accepted 12.8.13; Edited by A Stephanou
} 
features and investigated its subcellular localization and its role in apoptosis induced by STS. Our results contribute useful information on the $p J A B 1$ gene and offer a potential molecular model for studying diseases related to hJAB1.

\section{Results}

Identification and analysis of basic characteristics of pJAB1. The full-length cDNA of pJAB1 with $1076 \mathrm{bp}$ was obtained (Figure 1a) and deposited in GenBank under accession number JX570667. The cDNA encodes a protein of 334 amino acids, whose molecular weight $(\mathrm{Mw})$ is about $37.57 \mathrm{kDa}$, with the calculated isoelectric point ( $\mathrm{pl}$ ) being 6.10. To determine the exon-intron structure of the $p J A B 1$ gene, we performed a blast search in the pig reference genomic sequence database (Sscrofa10.2) using the pJAB1 cDNA sequence. A 17428 -bp sequence covering the entire pJAB1 gene was retrieved (GenBankID: NW 003610083.1). The alignment of the genomic sequence with the $p J A B 1$ cDNA sequence revealed that the exonic sequences matched $100 \%$ and there were a total of 8 exons ranging from 66 to 235 nucleotides, and all the observed splice acceptor and donor sites were in accordance with the GT-AG rule. The exon-intron structure is presented in Figure $1 \mathrm{~b}$. The secondary structure analysis showed that PJAB1 contained three conservative domains: chromo, MPN and DEAD domains (Figure 1c). The alignment of the protein sequence with the BLAST program on the NCBI website showed that pJAB1 gene shares 100, 99, 99, 98, 97, 96, 81 and 75\% identities with homologous genes of human, mouse, cattle, chicken, African clawed frog, zebrafish, Florida carpenter ant, and fruit fly, respectively. Because of the $100 \%$ similarity at the amino acid level between pJAB1 and hJAB1, we next made an alignment of the nucleotide sequences of these two species. As shown in Figure 1d, the $p J A B 1$ sequence differs by 47 nucleotides from that of $h J A B 1$. Moreover, as shown in Figure 2, despite some differences existing at amino acid level, the tertiary structure of pJAB1 was highly similar to its counterparts in other species, and the important structural amino acids were conserved in the same spatial position. In addition, subcellular localization prediction showed that pJAB1 may be a cytoplasmic protein.

Analysis of proteins interacting with pJAB1. The result of a protein-protein interaction analysis showed that pJAB1 could interact with several proteins, including COP9 constitutive photomorphogenic homolog subunit 2 (COPS2), COPS3, COPS4, COPS7A, tumor protein p53 (TP53), proteasome 26S subunit non-ATPase 6 (PSMD6), PSMD12, growth factor augmenter of liver regeneration (GFER), ring finger protein 139 (RNF139) and a protein with no annotation (Figure 3a). These identified proteins were sorted by the
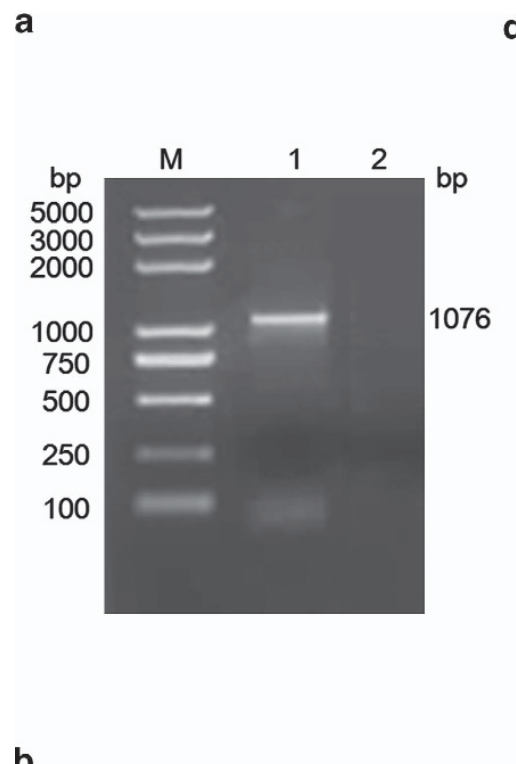
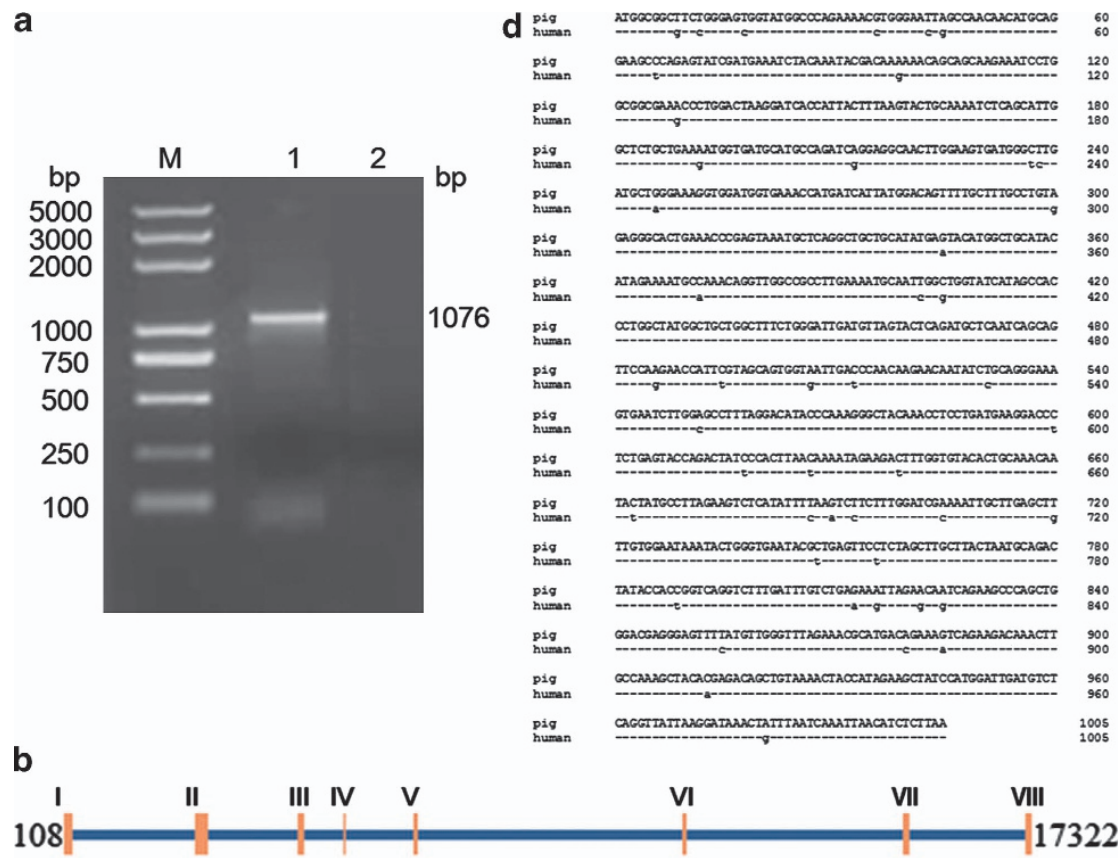

C

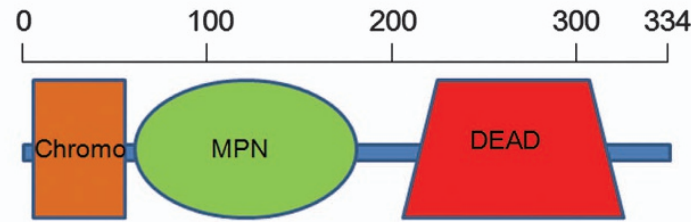

Figure 1 Basic characteristics of pJAB1. (a) The PCR result of the pJAB1 gene. M, 5000 bp DNA marker; 1, the pJAB1 gene; 2, blank control. (b) The exon-intron structure. Eight exons were found in the cDNA sequence. (c) The secondary structure showing chromo, MPN and DEAD domains in the pJAB1 protein. (d) Comparison of the nucleotide sequences of pJAB1 and hJAB1. Short bars represent the identical nucleotides among the two sequences. Different regions were indicated by lowercase letters 


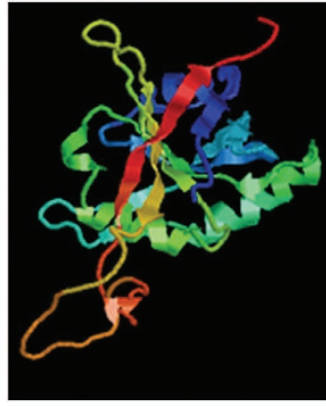

pig

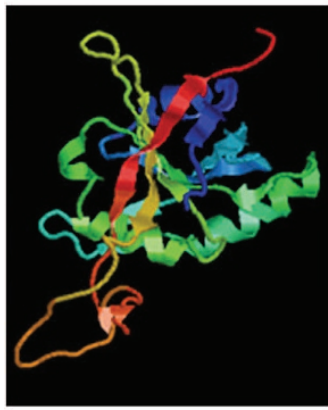

cattle

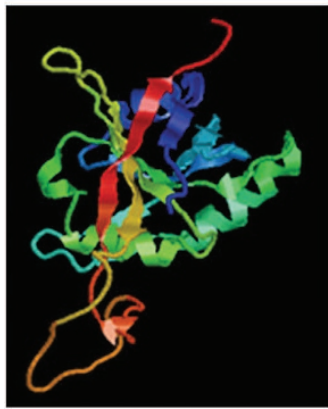

zebra fish

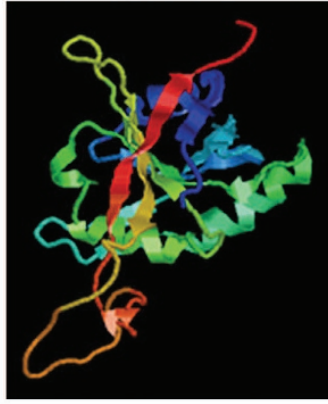

human

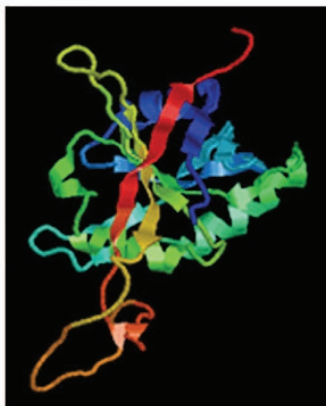

chicken

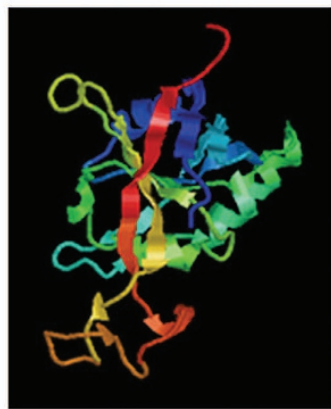

fruit fly

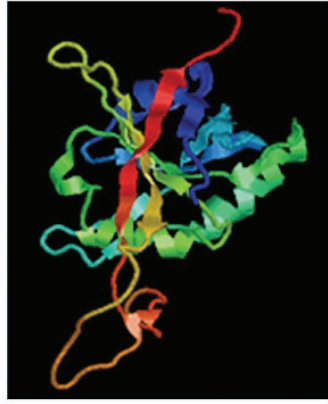

mouse

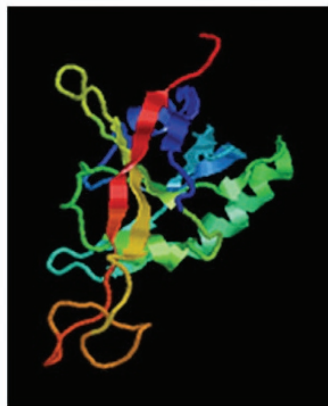

frog

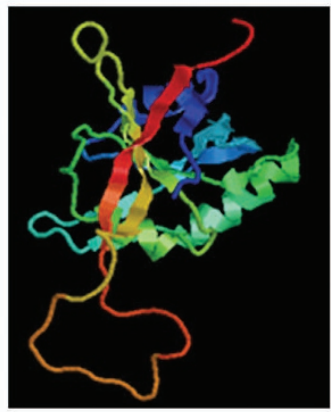

ant

Figure 2 The tertiary structures of pJAB1 and other JAB1 proteins. Different colors represent different motifs or domains

enrichment of Gene Ontology (GO) categories based on the UniProt database. These proteins mainly clustered into functional groups: cellular protein metabolic process, protein modification process, macromolecule modification, protein modification by small protein conjugation or removal, negative regulation of metabolic process, cullin deneddylation, signal transduction, negative regulation of G1/S transition of mitotic cell cycle, mitotic G1 DNA damage checkpoint, positive regulation of cell cycle arrest, cycle phase transition, regulation of cellular amino acid metabolic process, regulation of ubiquitin-protein ligase activity involved in mitotic cell cycle, antigen processing and presentation, and so on (Figure 3b).

JAB1 is highly conserved among different species. To evaluate the evolutionary relationship of pJAB1 with homologs from other species, we constructed a phylogenetic tree using the MEGA4 software. ${ }^{36}$ As shown in Figure 4 , these proteins formed two distinct clusters in this phylogenetic analysis: the first was the vertebrate cluster that consisted of the mammalian, he reptilian, the aves, the amphibia and the pisces, whereas the second was the invertebrate cluster that contained the arthopoda. The vertebrate cluster was then divided into two major clades. One clade contained the mammalian, the reptilia and the aves, and the other included the amphibia and the pisces. Not surprisingly, the mouse and the rat JAB1 proteins were sisters in their own clade. Similarly, the porcine and the human proteins formed a separate clade, together with the cattle protein. This suggests that hJAB1 is more closely related to the pJAB1 than to rodent JAB1 proteins. Within the invertebrate cluster, the fruit fly and the Glossina morsitans morsitans appeared as a sister group together with the southern house mosquito in the first clade, whereas the Florida carpenter ant and the Panamanian leafcutter ant emerged as a sister group together with the Jerdon's jumping ant, the Papilio xuthus and the pea aphid in the second clade of the phylogenetic tree. 


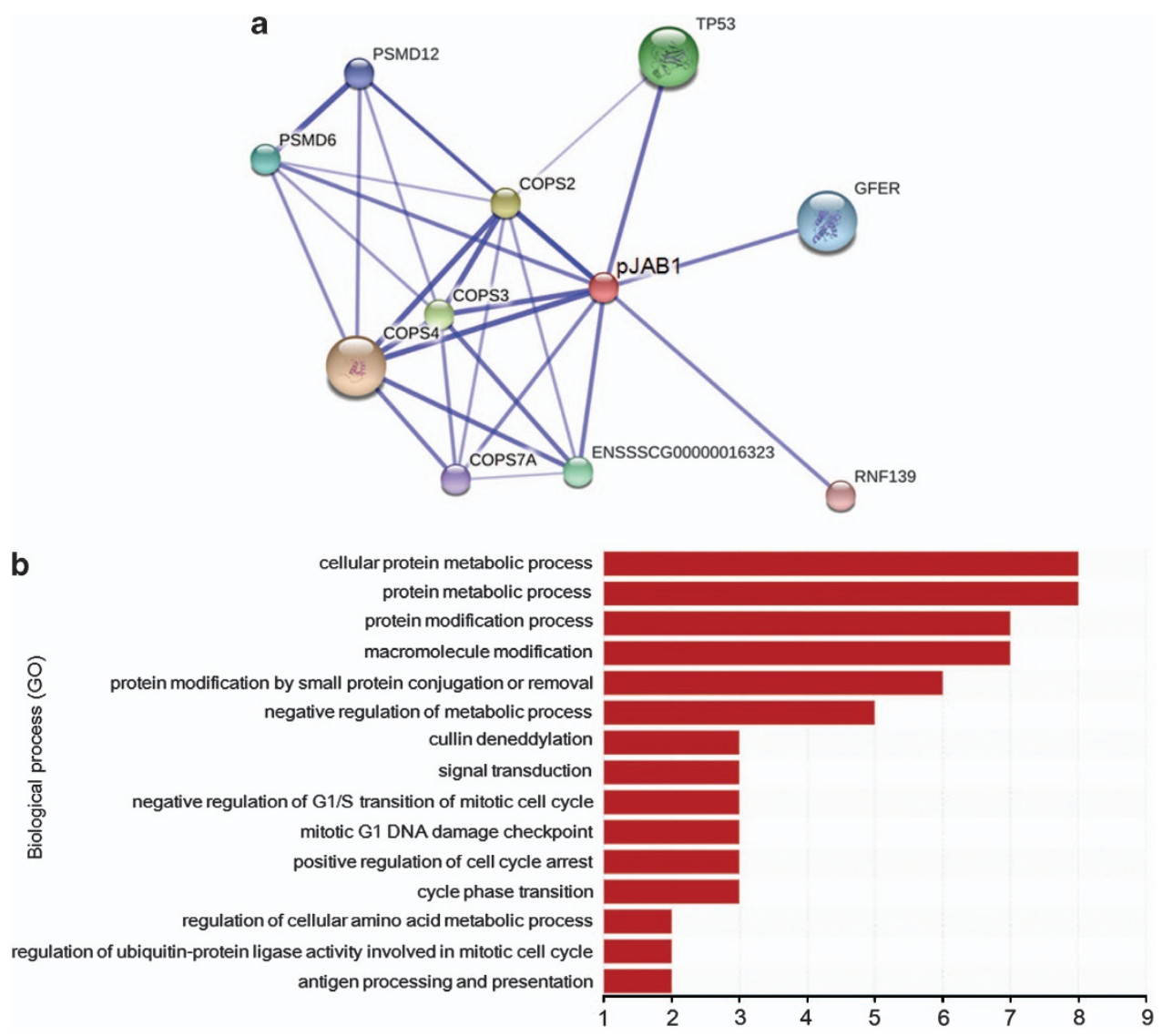

Figure 3 The network of proteins interacting with pJAB1 and functional classification of these proteins. (a) Proteins interacting with pJAB1 were identified using the STRING 9.1 software. Core protein pJAB1 was presented as red circle and its interactors were presented as circle of other colors. Stronger associations were represented by thicker lines. (b) Functional classification of these identified proteins was performed according to GO biological processes. These proteins were sorted by the enrichment of $\mathrm{GO}$ categories. The vertical axis is the GO category and the horizontal axis is the enrichment of GO

pJAB1 is universally expressed in porcine tissues. The spatial expression of the pJAB1 mRNA was examined by quantitative real-time RT-PCR in RNA samples isolated from different porcine tissues. The obtained results revealed differential expression of pJAB1 mRNA in different porcine tissues. As shown in Figure 5, pJAB1 transcripts were detected in all examined organs and tissues, with the highest levels in the heart and the lowest levels in the lung. The difference in the level of $p J A B 1$ transcripts between these two tissues exhibited more than 16-fold. A medium expression level was observed in the liver, tonsil and kidney tissues, whereas most other porcine tissues exhibited a low pJAB1 transcript level.

GFP-pJAB1 fusion protein distributes specifically in the cytoplasm. For convenient detection of the PJAB1 protein expression, the recombinant expressing vector pEGFPpJAB1 was generated by subcloning the pJAB1 cDNA into the pEGFP-C1 vector (BD Biosciences Clontech, San Jose, CA, USA). The expression of GFP-pJAB1 or GFP proteins in the established swine umbilical vein endothelial cell line (SUVEC) and porcine kidney-15 cell line (PK15) transfected with pEGFP-pJAB1 or pEGFP-C1 vectors was analyzed by inverted fluorescence microscopy (Figure $6 \mathrm{a}$ ) and western blot analysis (Figure 6b). Fluorescence signals from
SUVEC/PK15 cells transiently expressing GFP-pJAB1 proteins were examined by confocal fluorescence microscopy. GFP-pJAB1 fusion proteins were found to be distributed specifically in the cytoplasm, whereas GFP proteins were detected throughout control cells (Figure 6c).

pJAB1 enhances apoptosis induced by STS. JAB1 activities positively and negatively affect a number of pathways, including integrin signaling, cell cycle control and apoptosis. ${ }^{5-8,37,38}$ To explore the role of pJAB1 in apoptosis induced by STS, we applied annexin V-PE/7-AAD staining in combination with flow cytometry (FCM) to differentiate between apoptosis and necrotic cell death. After incubation of SUVEC cells transiently expressing GFP-pJAB1 or GFP with 10-1000 nM STS for $24 \mathrm{~h}$, we co-incubated the cells with annexin V-PE as an early marker for phosphatidylserine externalization at the cell membrane. Early apoptotic events are located in the lower right quadrant of the FCM diagrams, whereas late apoptosis is found in the upper right quadrant (Figure 7a). Early apoptotic events revealed a significant increase in apoptosis when cells transiently expressing GFP-pJAB1 were compared with cells expressing GFP control over a broad range of STS doses (Figure 7b; $P=0.009$ for $10 \mathrm{nM}$ STS; $P=0.02$ for $100 \mathrm{nM}$ STS; $P=0.001$ for $1000 \mathrm{nM}$ STS). 


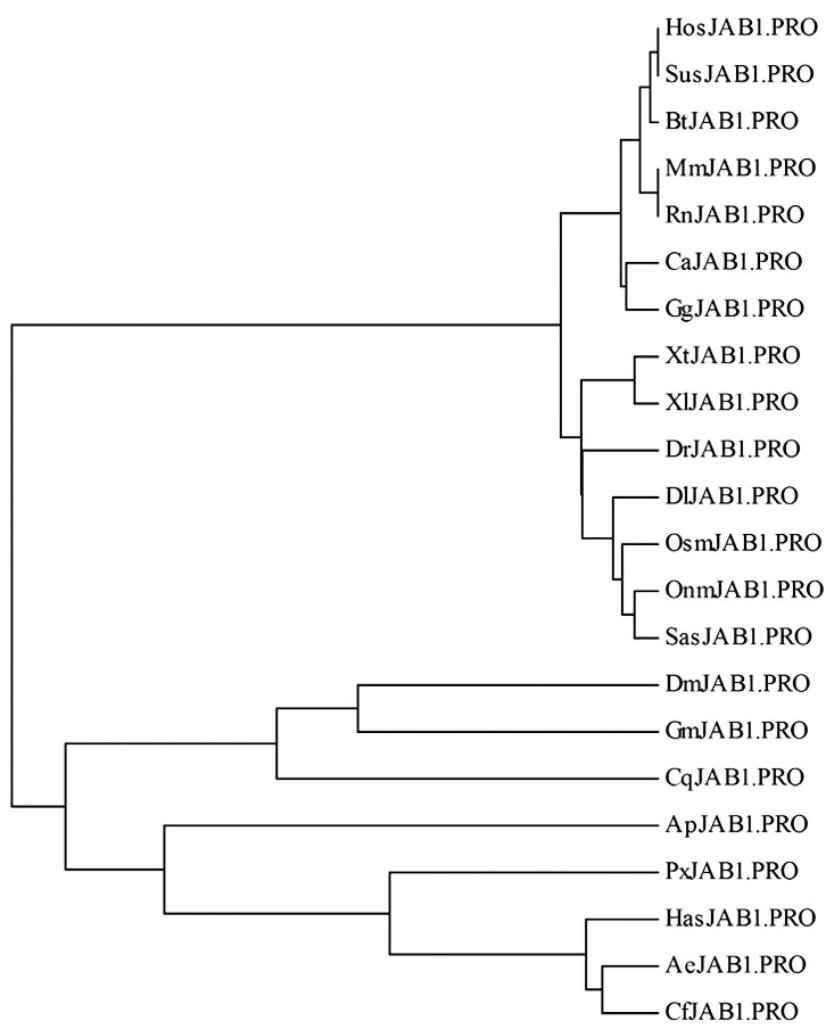

$\begin{array}{ccccccc}1 & 1 & 1 & 1 & 1 & 1 & 1 \\ 0.12 & 0.10 & 0.08 & 0.06 & 0.04 & 0.02 & 0.00\end{array}$

Figure 4 Evolutionary relationships of JAB1 proteins from 22 species. The phylogenetic tree was drawn using the MEGA4 software and the neighbor-joining method. The optimal tree with the sum of branch length $=0.79058468$ was shown. The abbreviations for species acronyms of the sequences used for construction of the phylogenetic tree are described as follows: Ae, Acromyrmex echinatior, Ap, Acyrthosiphon pisum; Bt, Bos taurus; $\mathrm{Ca}$, Crotalus adamanteus; $\mathrm{Cf}$, Camponotus floridanus; $\mathrm{Cq}$, Culex quinquefasciatus; Dr, Danio rerio; DI, Dicentrarchus labrax; Dm, Drosophila melanogaster, Gg, Gallus gallus; Gm, Glossina morsitans morsitans; Has, Harpegnathos saltator, Hos, Homo sapiens; мm, Mus musculus; Onm, Oncorhynchus mykiss; Osm, Osmerus mordax; Px, Papilio xuthus; Rn, Rattus norvegicus; Sas, Salmo salar, Sus, Sus scrofa; Xt, Xenopus (Silurana) tropicalis; $\mathrm{Xl}$, Xenopus laevis

Activation of caspase-9 and caspase-3, but not caspase-8, is involved in apoptosis induced by STS. In the present study, we explored the possible mechanisms of apoptosis induced by STS in SUVEC cells. As caspases are known to have pivotal roles in various apoptotic signaling pathways, we measured activities of initiator caspases (caspase-8 and caspase-9) and effector caspase (caspase-3) in STS-treated cells using the colorimetric assay kits. As shown in Figure 7c, exposure of SUVEC cells transiently expressing GFP-pJAB1 proteins to 100 nM STS significantly increased the activities of caspases- 9 and caspase- 3 in a time-dependent manner but had no effects on the activity of caspase- 8 during the treated period. We also investigated the effect of pJAB1 on the activation of caspase- 9 and caspase-3. As shown in Figure 7d, exposure of SUVEC cells transiently expressing GFP-pJAB1 proteins to $100 \mathrm{nM}$ STS for $6 \mathrm{~h}$ significantly increased the activation of caspase- 9 and caspase-3 compared with cells transiently expressing GFP

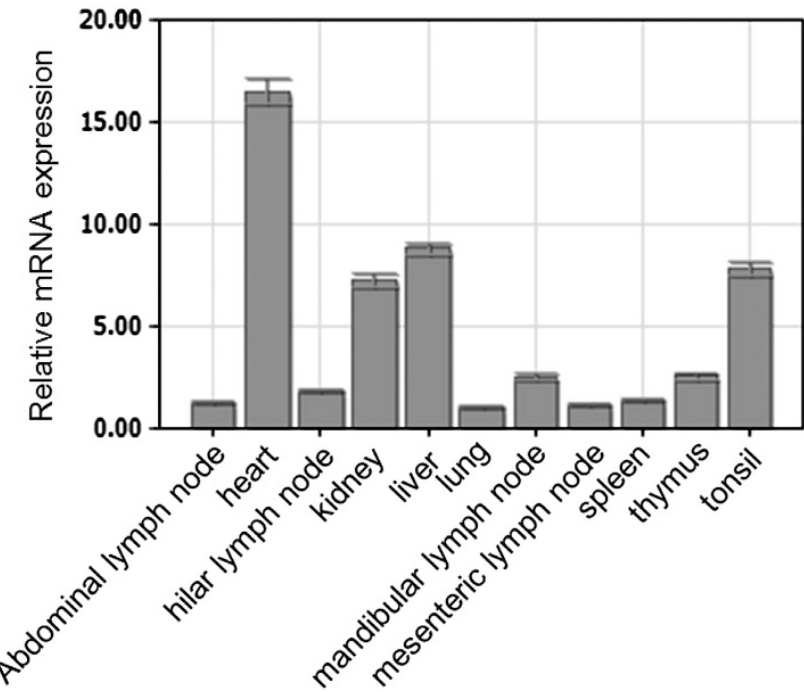

Figure 5 Spatial expression analysis of pJAB1 mRNA by real-time PCR. Mean mRNA levels in 11 tissues were analyzed. The values were normalized to endogenous $\beta$-actin expression. All experiments were performed in triplicate and data are expressed as means \pm s.d. $(n=3)$. Error bars represent s.d. of replicate data points

proteins with the same treatment. The activation of caspases induced by STS was further confirmed by western blot analysis. As shown in Figure 7e, treatment of SUVEC cells transiently expressing GFP-pJAB1 proteins with 100 nMSTS led to increased levels of cleaved caspase- 9 and caspase-3. In contrast, the cleavage of procaspase-8 was not observed in this study.

\section{Discussion}

In the present study, we cloned and characterized the $p J A B 1$ gene. Sequence analysis showed that the pJAB1 protein consisted of 334 amino acids, which was absolutely consistent with the hJAB1 protein, ${ }^{39}$ even though the nucleotide sequence of $p J A B 1$ differs by 47 nucleotides from that of hJAB1 (Figure 1d). The protein sequence of pJAB1 shows a high degree of conservation across species, especially in the MPN domain (Figure 1c). This is not surprising, as this domain is extremely important for JAB1 function. In addition, the tertiary structure of pJAB1 protein was highly similar with those of other species (Figure 2) and pJAB1 was clustered into the same clades with hJAB1 in the phylogenetic tree (Figure 4). Taken together, these results indicate that hJAB1 is evolutionarily closer to pJAB1 than to the rodent JAB1 proteins. It is therefore possible that pig could be more valuable as a model to study the function of JAB1 than the traditionally used mouse or rat models. Furthermore, the high conservation of JAB1 in biological evolution illustrates its importance in vivo.

As shown in Figure $3 a$, pJAB1 could interact with several proteins. Among these proteins, JAB1, COPS2, COPS3, COPS4 and COPS7A are members of the CSN complex; ${ }^{10}$ therefore, interactions of pJAB1 with COPS2, COPS3 and COPS4 are easy to understand and may be involved in multiple functions of the CSN complex. In human, TP53 is a 

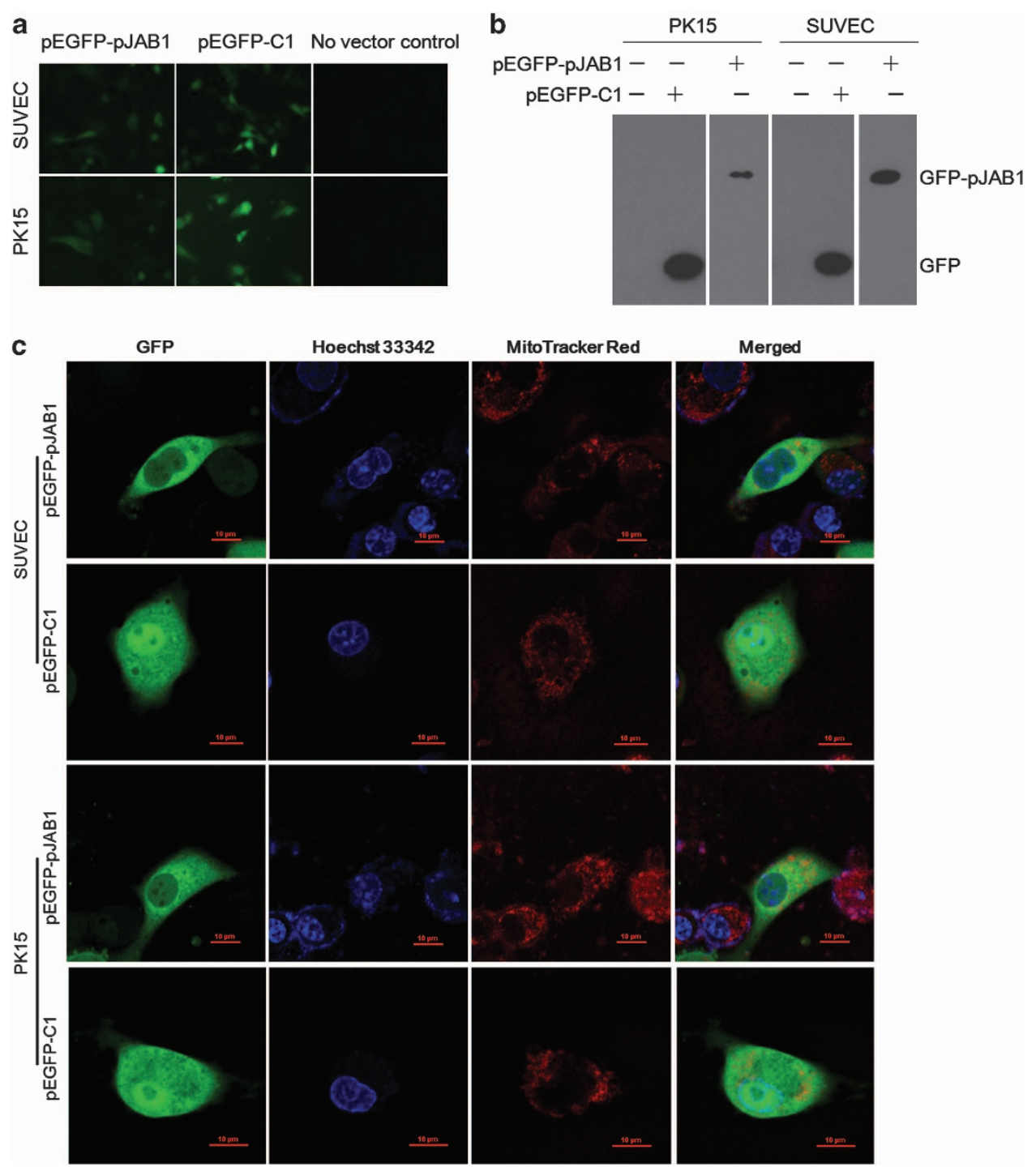

Figure 6 Detection and subcellular localization of the GFP-pJAB1 fusion protein transiently expressed in SUVEC/PK15 cells. (a) Analysis of GFP-pJAB1 or GFP protein expression by inverted fluorescence microscopy. (b) Western blot analysis of cellular proteins isolated from various cell lines using anti-GFP-tag antibody. (c) Confocal microscopy images of SUVEC/PK15 cells expressing GFP-pJAB1 or GFP proteins. All of the cell lines were stained by Hoechst 33342 and MitoTracker Red CMXRos. Merged images showed colocalization of GFP and GFP-pJAB1 in the mitochondrion. Scale bar, $10 \mu \mathrm{m}$ for all the Figures

tumor suppressor and JAB1 can enhance MDM2-mediated TP53 ubiquitination by mediating the nuclear export and cytoplasmic degradation of TP53. ${ }^{40}$ Thus, the interaction of pJAB1 with TP53 can make a contribution to studying tumorigenesis. PSMD6 and PSMD12 are the 26S subunits of proteasome, the interaction of pJAB1 with which indicates that pJAB1 may have a role in protein degradation. This is consistent with the report of hJAB1. For instance, hJAB1 induce degradation of several proteins such as Smad4, ${ }^{7}$ Smad7, ${ }^{41}$ TP53 (Oh et al. ${ }^{40}$ ) and p27. ${ }^{42}$ pJAB1 also interacts with GFER, a growth factor. This interaction suggests that pJAB1 can contribute to the cell proliferation. RNF139, a potential tumor suppressor gene, also is a membranebound E3 ubiquitin ligase that inhibits the growth of cells in a ubiquitylation-dependent manner. ${ }^{43,44}$ The interaction of pJAB1 with RNF139 suggests that pJAB1 has roles in tumor suppression and regulation of cell growth. Functions of these proteins mentioned above were consistent with the result of GO analysis (Figure $3 b$ ). Taken together, we can hypothesize that PJAB1 may be involved in tumorigenesis, protein degradation and cell proliferation. However, all of these hypotheses need to be proved by future experiments.

By quantitative real-time RT-PCR analysis, the tissue distribution of the pJAB1 transcripts was determined (Figure 5). Consistent with the expression pattern obtained for mouse JAB1, ${ }^{45}$ pJAB1 mRNA was detected in all the examined tissues. However, the difference between the expression patterns of these two species still exists. For instance, the highest level of pJAB1 transcripts was detected in the heart, whereas the highest level of mouse JAB1 transcripts was detected in the testis, ${ }^{45}$ which suggests that JAB1 may also have different roles in different species. 
a
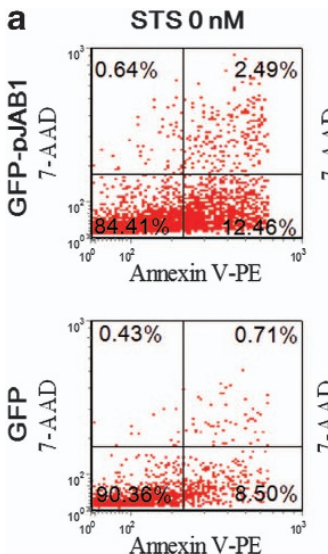

STS $10 \mathrm{nM}$

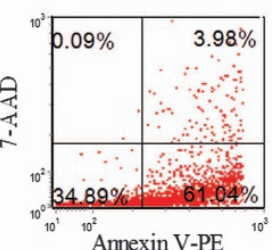

Annexin V-PE

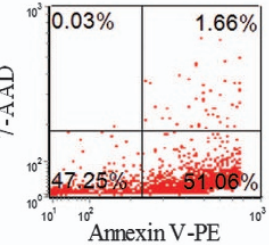

STS $100 \mathrm{nM}$

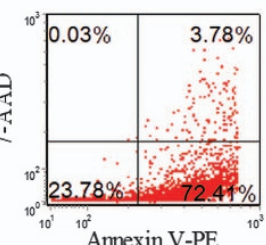

${ }^{10^{2}}$ Anmexin V-PE

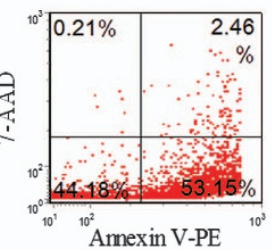

STS $1000 \mathrm{nM}$

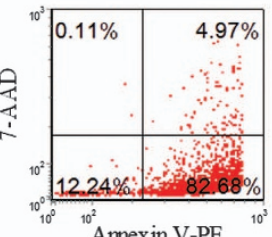

${ }^{10^{2}}$ Annexin V-PE

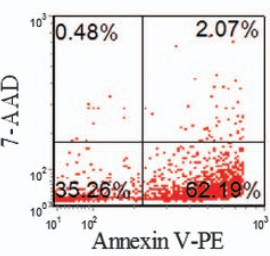

Annexin V-PE
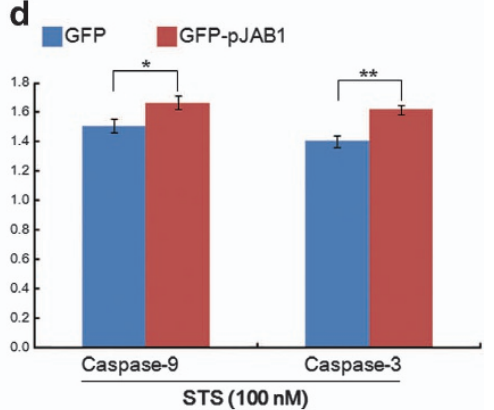

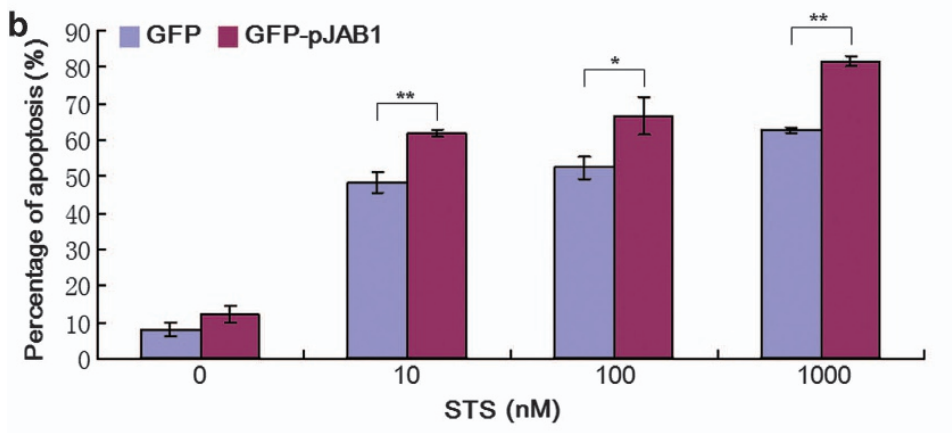

e

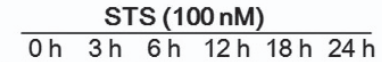

pro-caspase-8

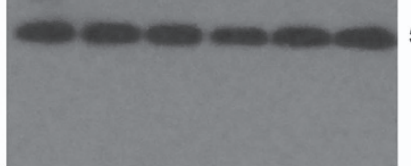

$55 \mathrm{kD}$

pro-caspase-9

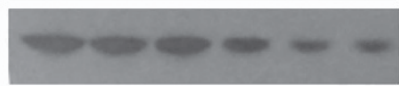

$46 \mathrm{kD}$

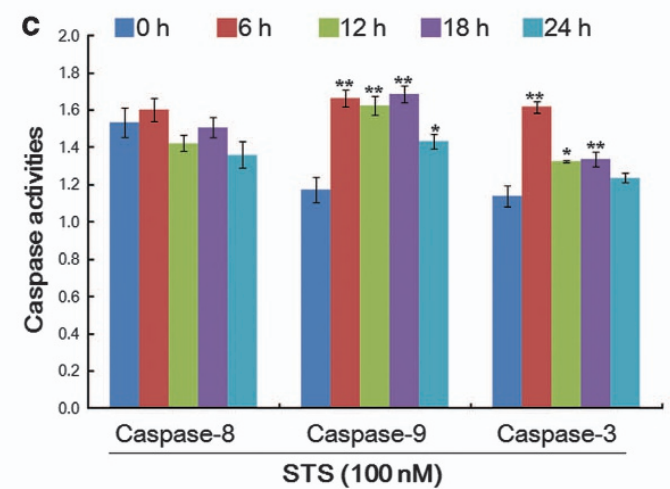

cleaved caspase- 9

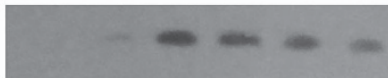

$35 \mathrm{kD}$

pro-caspase-3

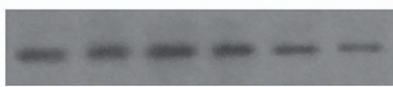

$32 \mathrm{kD}$

cleaved caspase-3

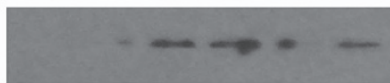

$17 \mathrm{kD}$

$\beta$-actin

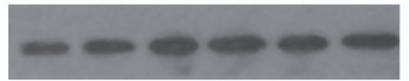

$43 \mathrm{kD}$

Figure 7 Quantification of apoptosis induced by STS and detection of caspase activity. (a) Representative FCM diagrams of apoptosis detection. SUVEC cells transiently expressing GFP-pJAB1 or GFP proteins were both treated with the corresponding STS doses for $24 \mathrm{~h}$. Next, apoptosis was detected with annexin V-PE or 7-AAD staining and FCM. (b) Quantification of apoptosis. All experiments were performed in triplicate and data of apoptosis are expressed as means \pm s.d. ( $n=3$ ). Error bars represent s.d. of replicate data points. ${ }^{*} P<0.05$, ${ }^{* *} P<0.01$ versus the control group (cells expressing GFP proteins). (c) Detection of caspase activity. SUVEC cells transiently expressing GFP-pJAB1 proteins were treated with $100 \mathrm{nM}$ STS for the indicated time. The activities of caspase-8, caspase- 9 and caspase-3 in these cells were measured using the colorimetric assay kits. All experiments were performed in triplicate and data are expressed as means \pm s.d. $(n=3)$. Error bars represent s.d. of replicate data points. ${ }^{\star} P<0.05,{ }^{* *} P<0.01$ versus the control group $(0 \mathrm{~h})$. (d) Effect of pJAB1 on caspase- 9 and caspase-3 activities in apoptosis induced by STS. SUVEC cells expressing GFP-pJAB1 proteins and those expressing GFP proteins were both treated with $100 \mathrm{nM}$ STS for $6 \mathrm{~h}$. The activities of caspase-9 and caspase- 3 in these cells were measured using the colorimetric assay kits. All experiments were performed in triplicate and data are expressed as means $\pm s . d$. ( $n=3$ ). Error bars represent s.d. of replicate data points. ${ }^{*} P<0.05,{ }^{*} P<0.01$ versus the control group (cells expressing GFP proteins). (e) Detection of the caspase activation by western blot. SUVEC cells transiently expressing GFP-pJAB1 proteins were treated with $100 \mathrm{nM}$ STS for indicated time. Whole-cell lysates were analyzed by western blot to detect pro- and cleaved caspase-8, caspase- 9 and caspase-3. The data shown are representative of three independent experiments

The pJAB1 protein has been found to localize specifically in the cytoplasm (Figure 6c), which is consistent with the result of subcellular localization prediction. Furthermore, the localization has not been affected by the different cell lines used. This result matches up with the localization of hJAB1. ${ }^{46}$ This result provides evidence for using pig as a model to study the function of hJAB1 and disease relevant with JAB1.
As mentioned previously, STS can induce apoptosis in several kinds of cancer cells, which suggests that STS may inhibit growth of tumors. Moreover, apoptosis induced by STS can be regulated by several proteins. These reports attract us to investigate the role of pJAB1 in apoptosis induced by STS. As shown in Figures $7 a$ and b, apoptosis was also induced by STS in SUVEC cells. Furthermore, the apoptosis was 
Table 1 Real-time PCR primer sequences

\begin{tabular}{|c|c|c|}
\hline \multirow[t]{2}{*}{ Genes } & \multicolumn{2}{|c|}{ Primer sequences $\left(5^{\prime} \rightarrow 3^{\prime}\right)$} \\
\hline & Forward & Reverse \\
\hline $\begin{array}{l}\text { pJAB1 } \\
\beta \text {-Actin }\end{array}$ & $\begin{array}{l}\text { AATCCTGGCGGCGAAAC } \\
\text { CAAGGACCTCTACGCCAACAC }\end{array}$ & $\begin{array}{l}\text { CATCAAGCCCATCACTTCCA } \\
\text { AGGATGGAGCCGCCGATC }\end{array}$ \\
\hline
\end{tabular}

significantly enhanced by pJAB1. This result suggests that pJAB1 may be involved in apoptosis induced by STS and elucidation of its action mechanism can provide useful information on model building for developing cancer therapy.

Next, we investigated the possible mechanisms of apoptosis induced by STS in SUVEC cells. Caspases have been well known to have pivotal roles in the execution of apoptosis. Two major caspases activation pathways, death receptor and mitochondrial pathways, have been well characterized. ${ }^{47}$ In the death receptor pathway, apoptosis occurs upon the activation of caspase-8 stimulated by death receptors in the cell surface, whereas in the mitochondrial pathway apoptosis is dependent on the activation of caspase- 9 resulting from the release of cytochrome $c$ from mitochondria to the cytosol. Caspase- 8 and caspase- 9 will then activate downstream caspases, in particular caspases-3, which is essential for the morphological change and DNA fragmentation associated with apoptosis.

In this study, we observed that the activation of caspase-9 and caspase-3 was involved in apoptosis induced by STS (Figures 7c and e), and that the pJAB1 significantly increased the activation of caspase-9 and caspase-3 (Figure 7d). In addition, we also proved that STS failed to activate the death receptor-mediated caspase- 8 pathway in this study (Figures 7c and e). Thus, our results demonstrate that the apoptosis induced by STS is at least partially dependent on the activation of caspases, especially capase-9 and caspase-3.

In conclusion, we cloned and characterized of the $p J A B 1$ gene, constructed a phylogenetic tree and analyzed its spatial expression patterns. Subcellular localization analysis showed GFP-pJAB1 fusion protein distributed specifically in the cytoplasm. The FCM analysis identified the enhancement of pJAB1 to apoptosis induced by STS. The caspase activity assay indicated that caspase- 9 and caspase- 3 were activated in apoptosis induced by STS, and that pJAB1 significantly increased the activation of caspase- 9 and caspase- 3 . It is obvious that we are far from unveiling the complete mechanisms of apoptosis enhanced by pJAB1 and that further studies need to be done to answer some questions. For instance, which are the protein partners interacting with pJAB1 and how does pJAB1 participate in apoptosis induced by STS? Ongoing work in our laboratory is addressing these issues. The fundamental data accumulated in this study provides a potential medical model for investigating the function of JAB1 and the diseases related with this gene.

\footnotetext{
Materials and Methods

Reagents. Dulbecco's modified Eagle's medium and fetal bovine serum were obtained from Hyclone (Beijing, China). STS was obtained from Sigma-Aldrich (St. Louis, MO, USA). Mouse monoclonal antibodies against caspase-8, caspase-9, caspase-3, GFP-tag and $\beta$-actin were purchased from Santa Cruz Biotechnology,
}

Inc. (Santa Cruz, CA, USA). Horseradish peroxidase (HRP)-conjugated goat antimouse secondary antibody was purchased from Sigma-Aldrich.

Animals and cells. The 1-month-old, large white pigs were housed and had been approved by the scientific ethical committee of the Northwest A\&F University before it was used in this study. PK15 was cultured in Dulbecco's modified Eagle's medium supplemented with $10 \%$ fetal bovine serum at $37^{\circ} \mathrm{C}$ under $5 \% \mathrm{CO}_{2}$, and SUVEC was cultured as previously described. ${ }^{48}$

Cloning of the pJAB1 gene and plasmid construction. First, we obtained a predicted nucleotide sequence encoding pJAB1 from NCBI. The primer pair (forward, 5'-TAGGGGCAACAACTTCTC- $3^{\prime}$ and reverse, 5'-TATTTTTCTCAT ACTGGC- $3^{\prime}$ ) was designed complementary to the forecasted sequence. Total RNA was extracted from the heart of 1-month-old, large white pig using TRIzol reagent (Invitrogen, Carlsbad, CA, USA). Extracted RNAs were subjected to RT-PCR using first-strand CDNA synthesis kit (Takara Bio., Dalian, China). Next, the CDNA was used to carry out PCR amplification using primers mentioned before. Amplified PCR products were electrophoresed on 1\% agarose gel. A band of $1076 \mathrm{bp}$ was observed, indicating it to be pJAB1. The positive PCR products were purified and were then cloned into pMD19-T vector (Takara Bio.) in order to be sequenced in both directions. CDNAs containing the ORF of pJAB1 without additional flanking sequences was generated by PCR with the following primer pair: forward, 5'-ATAGATCTATGGCGGCTTCTGGGAG-3' and reverse, $5^{\prime}$-TAGG GCCCTTAAGAGATGTTAATTTG- $3^{\prime}$. The resulting PCR products were digested with restriction endonucleases and subcloned into the $B g \| l$ and Apal sites of pEGFP-C1 vector (BD Biosciences Clontech). The recombinant vector was named pEGFP-pJAB1.

Bioinformatics analysis of sequence and structure. Initially, the cDNA sequence and corresponding genomic sequence were analyzed using the Spidey software (http://www.ncbi.nlm.nih.gov/spidey) in order to establish the exon-intron organization. Next, the mRNA sequence was subjected into the openreading frame (ORF) finder software (http://www.ncbi.nlm.nih.gov/gorf/gorf.html) to find the ORF and to acquire the amino acid sequence. The amino acid sequence was aligned using the protein blast software (http://blast.ncbi.nlm.nih.gov/Blast.cgi) and the DNAMAN 6.0.40 (LynnonBiosoft) software (http://lynnon-biosoft-dnaman-eng. software.informer.com/). The theoretical $\mathrm{pl}$ and $\mathrm{Mw}$ of the $\mathrm{pJAB} 1$ protein was computed using the Compute $\mathrm{pl} / \mathrm{Mw}$ Tool (http://web.expasy.org/compute_pi/). The conservative domains were predicted using the SBASE web tool (http://hydra.icgeb.trieste.it/sbase/sbase.php?sec=analyse\&sub=predict). The tertiary structures of JAB1 proteins from pig, human, mouse, cattle, chicken, frog, zebrafish, fruit fly and ant were determined using the $\mathrm{CPH}$ models 3.2 Server based on profileprofile alignment guided by secondary structure and exposure predictions, ${ }^{49}$ then visualized and manipulated using the RasWin Molecular Graphics Program (RasMol, version 2.7.4.2) software package (http://www.rasmol.org). The subcellular localization of the pJAB1 protein was predicted using the protein localization software (http://chemdata.shu.edu.cn/subcell/subcell4.jsp). Protein-protein interactions of pJAB1 were analyzed using the STRING (version 9.1) software. ${ }^{50} \mathrm{GO}$ analysis of proteins interacting with pJAB1 was carried out using the GO Term Enrichment tool in AmiGO (http://amigo.geneontology.org/cgi-bin/amigo/term_enrichment?session_id), the bar chart of which was constructed using metasee software. ${ }^{51}$ Because of the insufficiency of porcine protein data and the high similarity of proteins between pig and human, human protein data were used in the GO analysis.

Phylogenetic and molecular evolutionary analysis. Phylogenetic and molecular evolutionary analyses were conducted using MEGA4 software. ${ }^{36}$ The JAB1 protein sequences from 22 different species, that is, Panamanian leafcutter ant, pea aphid, cattle, Florida carpenter ant, eastern diamondback rattlesnake, southern house mosquito, zebrafish, European seabass, fruit fly, chicken, Glossina morsitans morsitans, Jerdon's jumping ant, human, house 
mouse, rainbow trout, rainbow smelt, Papilio xuthus, Norway rat, Atlantic salmon, western clawed frog, African clawed frog and pig were derived from Genbank (Genbank Nos. EGl68489, NP_001156462, NP_001179068, EFN60285, AFJ49753, XP_001862398, NP_957019, CBN81931, NP_477442, NP_001034400, ADD19140, EFN79117, NP_006828, NP_038743, NP_00115 8520, AC009632, BAM18530, NP_001020866, ACl68431, NP_989109, NP_001086291 and JX570667, respectively). The evolutionary history was inferred using the neighbor-joining method. ${ }^{52}$ The percentage of replicate trees in which the associated taxa clustered together in the bootstrap test (500 replicates) was shown next to the branches. ${ }^{53}$ The phylogenetic tree was linearized assuming equal evolutionary rates in all lineages. ${ }^{54}$ The tree was drawn to scale, with branch lengths in the same units as those of the evolutionary distances used to infer the phylogenetic tree. The evolutionary distances were computed using the Poisson correction method ${ }^{55}$ and were in the units of the number of amino acid substitutions per site. All positions containing gaps and missing data were eliminated from the data set. There were a total of 324 positions in the final data set.

Real-time PCR analysis of spatial expression patterns. Quantitative real-time RT-PCR was carried out in a Bio-Rad iQ 5 machine (Bio-Rad, Hercules, CA, USA) for analyzing tissue distribution of pJAB1. DNase-treated total RNA $(300 \mathrm{ng}$ ) was reverse transcribed and amplified in the same reaction tube using FastStart Universal SYBR Green Master (ROX; Roche Diagnostics GmbH, Mannheim, Germany), according to the manufacturer's instructions. The samples were run in triplicate in a total volume of $20 \mu \mathrm{l}$. The $2^{-\Delta \Delta \mathrm{Ct}}$ method was used to calculate relative gene expression. The $\beta$-actin gene, one of the housekeeping genes, was selected as an internal control for normalization, because it was consistently expressed in all tissues of this assay. The primers used are described in Table 1.

Transfection and intracellular distribution of PJAB1 protein. To determine the subcellular localization of pJAB1 protein, SUVEC/PK15 cells were cultured in six-well plates for $24 \mathrm{~h}$ as previously described, and then in medium without serum for $1 \mathrm{~h}$. The cells were transfected with $4 \mu \mathrm{g}$ of the pEGFP-pJAB1 plasmids combined with Lipofectamine2000 (Invitrogen), according to the manufacturer's instructions. Cells were transfected with pEGFP-C1 vectors as a negative control. GFP-pJAB1 or GFP proteins expressed in transfected cells were analyzed by inverted fluorescence microscopy and western blot analysis. About $36 \mathrm{~h}$ after transfection, cells were washed with PBS and then incubated at $37^{\circ} \mathrm{C}$ for $30 \mathrm{~min}$ in growth medium containing $50 \mathrm{nM}$ MitoTracker Red CMXRos (Invitrogen) for mitochondrial labeling. After being washed with PBS, the cells were incubated at $37^{\circ} \mathrm{C}$ for $20 \mathrm{~min}$ with $6 \mu \mathrm{g} / \mathrm{ml}$ Hoechst 33342 (Invitrogen). Then the cells were analyzed by A1R MP Confocal Microscope (Nikon Instruments, Tokyo, Japan). The results were processed with the software NIS Viewer (Nikon Instruments).

Western blot analysis. Protein samples were extracted from indicated cells using lysis buffer $(50 \mathrm{mM}$ Tris- $\mathrm{HCl}, 5 \mathrm{mM}$ EDTA, $150 \mathrm{mM} \mathrm{NaCl}, 0.1 \% \mathrm{NP}-40,0.5 \%$ deoxycholic acid, $1 \mathrm{mM}$ sodium orthovanadate, $100 \mu \mathrm{g} / \mathrm{ml}$ PMSF and protease inhibitors). The protein concentration was determined using the BCA Protein Assay Kit (Pierce, Rockford, IL, USA). Equivalent amounts of protein samples were uploaded and separated by SDS-PAGE and then transferred to a polyvinylidene fluoride membrane (Millipore, Billerica, MA, USA). The membrane was blocked with blocking buffer ( $5 \%$ skimmed milk in Tris-HCl buffered saline) and then incubated with indicated primary antibodies overnight at $4{ }^{\circ} \mathrm{C}$, followed by HRP-conjugated secondary antibodies at room temperature for $1 \mathrm{~h}$. The protein bands were visualized by Luminata Classico Western HRP Substrate as per the manufacturer's instructions (Millipore).

Analysis of apoptosis by flow cytometry. To study whether the pJAB1 gene has any function related to apoptosis, we detected the apoptosis of SUVEC cells transiently expressing GFP-pJAB1 induced by STS. The FCM system CyFlow Cube6 (Partec GmbH, Munster, Germany) and annexin V-PE/7-AAD kit (BD Bioscience, Rockville, MD, USA) were used according to the manufacturer's instructions. SUVEC cells transiently expressing GFP-pJAB1 or GFP were plated in six-well plates at a concentration of $2.5 \times 10^{5}$ cells per well $(2 \mathrm{ml} /$ well) and were cultured as previously described. When about $80 \%$ confluence was observed, the medium was replaced with fresh challenge media supplemented with 10-1000 nM STS. Cells were collected $24 \mathrm{~h}$ after treatment. After being washed with D-Hanks, the cells were detached with trypsin and $5 \times 10^{5}$ cells of each group were resuspended in binding buffer and incubated with annexin V-PE/7-AAD for $15 \mathrm{~min}$ at $25^{\circ} \mathrm{C}$. Dead cells and debris were excluded by selective gating based on electronic cell volume. About 20000 events were collected from each sample. By using dual-parameter staining, early apoptosis was quantified, using the CyView8.5 software (Partec). In preliminary experiments, unstained mediumtreated cells were used to define living cells (lower left quadrant). Cells treated with $\mathrm{H}_{2} \mathrm{O}_{2}$ as a positive control were singly stained with annexin V-PE/7-AAD to define, respectively, the lower right and upper left quadrants.

Caspase activity assay. Activities of caspase-8, caspase- 9 and caspase- 3 were measured by colorimetric assay kits (BioVision, Inc., Mountain View, CA, USA) according to the manufacturer's instructions. Briefly, cells were collected and lysed on ice and protein concentration was measured using BCA Protein Assay Reagent (Pierce), then $200 \mu \mathrm{g}$ of the protein was incubated with each caspase substrate at $37^{\circ} \mathrm{C}$ in a microplate for $4 \mathrm{~h}$. The resultant color was read at $405 \mathrm{~nm}$ in a Thermo Scientific Multiskan FC microplate photometer (Thermo Fisher Scientific Inc., Vantaa, Finland).

Statistical analysis. Calculation of means and s.d., and statistical analysis were performed with SPSS 17.0 (IBM SPSS; http://www-01.ibm.com/software/ analytics/spss/). Differences between test and control groups were assessed using independent samples test, with $P$-value below 0.05 considered statistically significant.

\section{Conflict of Interest}

The authors declare no conflict of interest.

Acknowledgements. This work was supported by the National Natural Science Foundation of China (grant no. 31072115) and a preparatory project sponsored by the National Ministry of Science and Technology of China of the First Batch in the Basic Research Category of the National Program of Science and Technology in the Field of Countryside 2011-2015 (Preparatory Project No. NC2010CD0178).

1. Claret FX, Hibi M, Dhut S, Toda T, Karin M. A new group of conserved coactivators that increase the specificity of AP-1 transcription factors. Nature 1996; 383: 453-457.

2. Seeger M, Kraft R, Ferrell K, Bech-Otschir D, Dumdey R, Schade R et al. A novel protein complex involved in signal transduction possessing similarities to $26 \mathrm{~S}$ proteasome subunits. FASEB J 1998; 12: 469-478.

3. Wei N, Tsuge T, Serino G, Dohmae N, Takio K, Matsui M et al. The COP9 complex is conserved between plants and mammals and is related to the $26 \mathrm{~S}$ proteasome regulatory complex. Curr Biol 1998; 8: 919-922.

4. Wei N, Deng XW. Making sense of the COP9 signalosome. A regulatory protein complex conserved from Arabidopsis to human. Trends Genet 1999; 15: 98-103.

5. Tomoda K, Kubota Y, Kato J. Degradation of the cyclin-dependent-kinase inhibitor p27Kip1 is instigated by Jab1. Nature 1999; 398: 160-165

6. Bech-Otschir D, Kraft R, Huang X, Henklein P, Kapelari B, Pollmann C et al. COPg signalosome-specific phosphorylation targets $p 53$ to degradation by the ubiquitin system. EMBO J 2001; 20: 1630-1639.

7. Wan M, Cao X, Wu Y, Bai S, Wu L, Shi X et al. Jab1 antagonizes TGF-beta signaling by inducing Smad4 degradation. EMBO J Rep 2002; 3: 171-176.

8. Oh W, Yang MR, Lee EW, Park KM, Pyo S, Yang JS et al. Jab1 mediates cytoplasmic localization and degradation of West Nile virus capsid protein. J Biol Chem 2006; 281: 30166-30174

9. Zhou J, Wan B, Li R, Gu X, Zhong Z, Wang Y et al. Jab1 interacts with brain-specific kinase 2 (BRSK2) and promotes its degradation in the ubiquitin-proteasome pathway. Biochem Biophys Res Commun 2012; 422: 647-652.

10. Chamovitz DA, Segal D. JAB1/CSN5 and the COP9 signalosome. A complex situation. EMBO Rep 2001; 2: 96-101.

11. Cope GA, Suh GS, Aravind L, Schwarz SE, Zipursky SL, Koonin EV et al. Role of predicted metalloprotease motif of Jab1/Csn5 in cleavage of Nedd8 from Cul1. Science 2002; 298 : 608-611.

12. Cope GA, Deshaies RJ. COP9 signalosome: a multifunctional regulator of SCF and other cullin-based ubiquitin ligases. Cell 2003; 114: 663-671.

13. Wolf DA, Zhou C, Wee S. The COP9 signalosome: an assembly and maintenance platform for cullin ubiquitin ligases? Nat Cell Biol 2003; 5: 1029-1033

14. Dubiel W. Resolving the CSN and CAND1 paradoxes. Mol Cell 2009; 35: 547-549.

15. Emberley ED, Niu Y, Leygue E, Tomes L, Gietz RD, Murphy LC et al. Psoriasin interacts with Jab1 and influences breast cancer progression. Cancer Res 2003; 63: 1954-1961. 
16. Li Y, Lu C, Xing G, Zhu Y, He F. Macrophage migration inhibitory factor directly interacts with hepatopoietin and regulates the proliferation of hepatoma cell. Exp Cell Res 2004; 300: 379-387.

17. Fukumoto A, Tomoda K, Yoneda-Kato N, Nakajima Y, Kato JY. Depletion of Jab1 inhibits proliferation of pancreatic cancer cell lines. FEBS Lett 2006; 580: 5836-5844.

18. Tamaoki T, Nakano H. Potent and specific inhibitors of protein kinase $\mathrm{C}$ of microbial origin. Biotechnology 1990; 8: 732-735.

19. Ballesta A, Lopez J, Popgeorgiev N, Gonzalo P, Doumic M, Gillet G. Data-driven modeling of SRC control on the mitochondrial pathway of apoptosis: implication for anticancer therapy optimization. PLoS Comput Biol 2013; 9: e1003011.

20. Simenc J, Lipnik-Stangelj M. Staurosporine induces different cell death forms in cultured rat astrocytes. Radiol Oncol 2012; 46: 312-320.

21. Hasegawa Y, Shimizu T, Takahashi N, Okada Y. The apoptotic volume decrease is an upstream event of MAP kinase activation during staurosporine-induced apoptosis in HeLa cells. Int J Mol Sci 2012; 13: 9363-9379.

22. Daudt DR, Yorio T. FKBP51 protects $661 \mathrm{w}$ cell culture from staurosporine-induced apoptosis. Mol Vis 2011; 17: 1172-1181.

23. Emanuelsson I, Norlin M. Protective effects of 27- and 24-hydroxycholesterol against staurosporine-induced cell death in undifferentiated neuroblastoma SH-SY5Y cells. Neurosci Lett 2012; 525: 44-48.

24. Zhao C, Yin P, Mei C, Li N, Yao W, Li X et al. Down-regulation of DNA methyltransferase $3 \mathrm{~B}$ in staurosporine-induced apoptosis and its mechanism in human hepatocarcinoma cell lines. Mol Cell Biochem 2013; 376: 111-119.

25. Yamaki K, Hong J, Hiraizumi K, Ahn JW, Zee O, Ohuchi K. Participation of various kinases in staurosporine induced apoptosis of RAW 264.7 cells. J Pharm Pharmacol 2002; 54: 1535-1544.

26. Li X, Li Z, Li N, Qi J, Fan K, Yin P et al. MAGl2 enhances the sensitivity of BEL-7404 human hepatocellular carcinoma cells to staurosporine-induced apoptosis by increasing PTEN stability. Int J Mol Med 2013; e-pub ahead of print 7 June 2013; doi:10.3892/jmm.2013.1411.

27. Shin JH, Kim HW, Rhyu IJ, Song KJ, Kee SH. Axin expression reduces staurosporine-induced mitochondria-mediated cell death in HeLa cells. Exp Cell Res 2012; 318: 2022-2033; e-pub ahead of print 7 June 2013; doi:10.3892/ijmm.2013.1411.

28. Cozzi E, Tucker AW, Langford GA, Pino-Chavez G, Wright L, O'Connell MJ et al. Characterization of pigs transgenic for human decay-accelerating factor. Transplantation 1997; 64: 1383-1392.

29. Ibrahim Z, Busch J, Awwad M, Wagner R, Wells K, Cooper DK. Selected physiologic compatibilities and incompatibilities between human and porcine organ systems. Xenotransplantation 2006; 13: 488-499.

30. Rogers CS, Abraham WM, Brogden KA, Engelhardt JF, Fisher JT, McCray PB Jr. et al. The porcine lung as a potential model for cystic fibrosis. Am J Physiol Lung Cell Mol Physiol 2008; 295: L240-L263.

31. Rogers CS, Stoltz DA, Meyerholz DK, Ostedgaard LS, Rokhlina T, Taft PJ et al. Disruption of the CFTR gene produces a model of cystic fibrosis in newborn pigs. Science 2008; 321 : 1837-1841.

32. Soerensen KE, Olsen HG, Skovgaard K, Wiinberg B, Nielsen OL, Leifsson PS et al. Disseminated intravascular coagulation in a novel porcine model of severe Staphylococcus aureus sepsis fulfills human clinical criteria. J Comp Pathol 2013; e-pub ahead of print 6 June 2013; pii: S0021-9975(13)00078-9; doi:10.1016/j.jcpa.2013.04.003

33. Czerski A, Bujok J, Gnus J, Hauzer W, Ratajczak K, Nowak M et al. Experimental methods of abdominal aortic aneurysm creation in swine as a large animal model. J Physiol Pharmacol 2013; 64: 185-192.

34. Nelson D, Porta C, Satterly S, Blair K, Johnson E, Inaba K et al. Physiology and cardiovascular effect of severe tension pneumothorax in a porcine model. J Surg Res 2013; 184: 450-457.

35. Flisikowska T, Kind A, Schnieke A. The new pig on the block: modelling cancer in pigs. Transgenic Res 2013; 22: 673-680.

36. Tamura K, Dudley J, Nei M, Kumar S. MEGA4: Molecular Evolutionary Genetics Analysis (MEGA) software version 4.0. Mol Biol Evol 2007; 24: 1596-1599.
37. Liu X, Pan Z, Zhang L, Sun $Q$, Wan J, Tian $C$ et al. JAB1 accelerates mitochondria apoptosis by interaction with proapoptotic BclGs. Cell Signal 2008; 20: 230-240.

38. Pan $\mathrm{Y}$, Zhang $\mathrm{Q}$, Tian $\mathrm{L}$, Wang $\mathrm{X}$, Fan $\mathrm{X}$, Zhang $\mathrm{H}$ et al. Jab1/CSN5 negatively regulates p27 and plays a role in the pathogenesis of nasopharyngeal carcinoma. Cancer Res 2012; 72: $1890-1900$

39. Shackleford TJ, Claret FX. JAB1/CSN5: a new player in cell cycle control and cancer. Cell Div 2010; 5: 26

40. Oh W, Lee EW, Sung YH, Yang MR, Ghim J, Lee HW et al. Jab1 induces the cytoplasmic localization and degradation of p53 in coordination with Hdm2. J Biol Chem 2006; 281: 17457-17465.

41. Kim BC, Lee HJ, Park SH, Lee SR, Karpova TS, McNally JG et al. Jab1/CSN5, a component of the COP9 signalosome, regulates transforming growth factor beta signaling by binding to Smad7 and promoting its degradation. Mol Cell Biol 2004; 24: 2251-2262.

42. Larrea MD, Wander SA, Slingerland JM. p27 as Jekyll and Hyde: regulation of cell cycle and cell motility. Cell Cycle 2009; 8: 3455-3461.

43. Gemmill RM, West JD, Boldog F, Tanaka N, Robinson LJ, Smith DI et al. The hereditary renal cell carcinoma 3;8 translocation fuses FHIT to a patched-related gene, TRC8. Proc Natl Acad Sci USA 1998; 95: 9572-9577.

44. Brauweiler A, Lorick KL, Lee JP, Tsai YC, Chan D, Weissman AM et al. RING-dependent tumor suppression and G2/M arrest induced by the TRC8 hereditary kidney cancer gene. Oncogene 2007; 26: 2263-2271

45. Bounpheng MA, Melnikova IN, Dodds SG, Chen H, Copeland NG Gilbert DJ et al. Characterization of the mouse JAB1 cDNA and protein. Gene 2000; 242: 41-50.

46. Lu C, Li Y, Zhao $Y$, Xing G, Tang F, Wang $Q$ et al. Intracrine hepatopoietin potentiates AP-1 activity through JAB1 independent of MAPK pathway. FASEB J 2002; 16: 90-92.

47. Ghobrial IM, Witzig TE, Adjei AA. Targeting apoptosis pathways in cancer therapy. CA Cancer J Clin 2005; 55: 178-194.

48. Hong HX, Zhang YM, Xu H, Su ZY, Sun P. Immortalization of swine umbilical vein endothelial cells with human telomerase reverse transcriptase. Mol Cell 2007; 24: 358-363.

49. Nielsen M, Lundegaard C, Lund O, Petersen TN. CPHmodels-3.0-remote homology modeling using structure-guided sequence profiles. Nucleic Acids Res 2010; 38(Web Server issue): W576-W581

50. Franceschini A, Szklarczyk D, Frankild S, Kuhn M, Simonovic M, Roth A et al. STRING v9.1: protein-protein interaction networks, with increased coverage and integration. Nucleic Acids Res 2013; 41(Database issue): D808-D815.

51. Song B, Su X, Xu J, Ning K. MetaSee: an interactive and extendable visualization toolbox for metagenomic sample analysis and comparison. PLOS One 2012; 7: e48998.

52. Saitou N, Nei M. The neighbor-joining method: a new method for reconstructing phylogenetic trees. Mol Biol Evol 1987; 4: 406-425.

53. Felsenstein J. Confidence limits on phylogenies: an approach using the bootstrap. Evolution 1985; 39: 783-791.

54. Takezaki N, Rzhetsky A, Nei M. Phylogenetic test of the molecular clock and linearized trees. Mol Biol Evol 1995; 12: 823-833.

55. Zuckerkandl E, Pauling L. Evolutionary divergence and convergence in proteins. Evol Genes Proteins 1965; 97: 166.

(c) (i) () Cell Death and Disease is an open-access journal published by Nature Publishing Group. This work is licensed under a Creative Commons Attribution-NonCommercialShareAlike 3.0 Unported License. To view a copy of this license, visit http://creativecommons.org/licenses/by-nc-sa/3.0/ 